\title{
Obstacle avoidance routing scheme through optimal sink movement for home monitoring and mobile robotic consumer devices
}

Article

Accepted Version

Chanak, P., Banerjee, I., Wang, J. and Sherratt, S. (2014) Obstacle avoidance routing scheme through optimal sink movement for home monitoring and mobile robotic consumer devices. IEEE Transactions on Consumer Electronics, 60 (4). pp. 596-604. ISSN 0098-3063 doi:

https://doi.org/10.1109/TCE.2014.7027292 Available at https://centaur.reading.ac.uk/39295/

It is advisable to refer to the publisher's version if you intend to cite from the work. See Guidance on citing.

To link to this article DOI: http://dx.doi.org/10.1109/TCE.2014.7027292

Publisher: IEEE

All outputs in CentAUR are protected by Intellectual Property Rights law, including copyright law. Copyright and IPR is retained by the creators or other copyright holders. Terms and conditions for use of this material are defined in the End User Agreement. 


\section{www.reading.ac.uk/centaur}

\section{CentAUR}

Central Archive at the University of Reading

Reading's research outputs online 


\section{Full Text of article}

Title: $\quad$ Obstacle Avoidance Routing Scheme through Optimal Sink Movement for Home Monitoring and Mobile Robotic Consumer Devices

Publication: IEEE Transactions on Consumer Electronics

Volume: $\quad 60$

Issue: $\quad 4$

pp.: $\quad 596-604$

URL: $\quad$ http://dx.doi.org/10.1109/TCE.2014.7027292

DOI: $\quad$ 10.1109/TCE.2014.7027292

Authors:

Prasenjit Chanak, Student Member, IEEE, Department of Information Technology, Indian Institute of Engineering Science and Technology, Shibpur, Howrah-711103, India (e-mail: prasenjit.chanak.2013@ieee.org, ibanerjee@it.becs.ac.in)

Indrajit Banerjee, Member, IEEE, Department of Information Technology, Indian Institute of Engineering Science and Technology, Shibpur, Howrah-711103, India (e-mail: prasenjit.chanak.2013@ieee.org, ibanerjee@it.becs.ac.in)

Jin Wang, Member, IEEE, School of Computer and Software, Jiangsu Engineering Center of Network Monitoring, Nanjing University of Information Science \& Technology, 210044, China (e-mail: wangjin@ nuist.edu.cn)

R. Simon Sherratt, Fellow, IEEE, School of Systems Engineering, University of Reading, Berkshire, RG6 6AY UK (e-mail: sherratt@ieee.org).

This work was supported partly by the Major Research Project under University Grants Commission (UGC), Govt. of India (F.No. 42-146/2013).

Abstract - In recent years, ZigBee has been proven to be an excellent solution to create scalable and flexible home automation networks. In a home automation network, consumer devices typically collect data from a home monitoring environment and then transmit the data to an end user through multi-hop communication without the need for any human intervention. However, due to the presence of typical obstacles in a home environment, error-free reception may not be possible, particularly for power constrained devices. A mobile sink based data transmission scheme can be one solution but obstacles create significant complexities for the sink movement path determination process. Therefore, an obstacle avoidance data routing scheme is of vital importance to the design of an efficient home automation system.

This paper presents a mobile sink based obstacle avoidance routing scheme for a home monitoring system. The mobile sink collects data by traversing through the obstacle avoidance path. Through ZigBee based hardware implementation and verification, the proposed scheme successfully transmits data through the obstacle avoidance path to improve network performance in terms of life span, energy consumption and reliability. The application of this work can be applied to a wide range of intelligent pervasive consumer products and services including robotic vacuum cleaners and personal security robots ${ }^{I}$.

Index Terms — Home Monitoring, Zigbee, Mobile Sink, Routing, Obstacle Detection. 


\section{Introduction}

Home automation networks are a significant application of Wireless Sensor Networks (WSN) where WSN and Consumer Electronics technology work together efficiently to construct a smart home [1]-[3]. In a home automation network, smart consumer sensor nodes collect data from monitoring field and then transmit the data to the Base Station (BS) through multi-hop communication without any human intervention [4]. Different types of physical obstacles may be present in and around the home environment, e.g. walls, furniture, human body, etc. These physical obstacles can hamper the data transmission path between sensor nodes and the BS. Therefore, data reception faults or data inconsistency faults have been occurring in home automation networks [5]. An obstacle avoidance data routing scheme can alleviate and minimize reception faults and improve the performance of the home monitoring network.

Recently, a mobile sink based data collection technique has become an important research topic in smart home network design [6]. The mobile sink based data collection scheme tremendously improves the network performance and lifetime. Distributed robotics and WSN have been working jointly in mobile sink based home automatic networks [7]. In the mobile sink based home automatic network [8], the mobile sink moves along different paths to collect data from deployed sensor nodes. After data collection, the mobile sink transmits data to the BS through either single-hop or multi-hop communication. Due to presence of obstacles within the home monitoring environment, finding the optimal sink movement path is a challenging issue. In existing mobile sink based home networks, the mobile sink randomly moves to a different location and collects data from static sensor nodes; however these techniques are unable to detect the presence of all obstacles within the monitoring environment. Therefore, these techniques are unable to calculate the optimal path under typical home environments that usually have obstacles.

The ZigBee standard has provided a solution for a scalable and flexible home automation network design due to its advantages in low power, low cost, and low communication overhead [9]. Kim et al. [10] proposed UPnP and multi-hop ZigBee networks for home automation systems. Kim et al. combined UPnP with multi-hop ZigBee network to design an efficient home network. Kim et al. [11] proposed another energy efficient traffic scheduling algorithm for IEEE 802.15.4 based home automation networks, where an efficient scheduling method was implemented to improve network lifetime. This technique used a multi-hop ZigBee network as well as single hop star topology creating an efficient scheduling scheme. However, the above techniques are unable to detect the presence of obstacles within the monitoring environment. Therefore, these techniques are unable to avoid data reception failures in the presence of obstacles. Besides, ZigBee based techniques only support static sink based data routing strategies.

This paper proposes a mobile sink based obstacle avoidance data routing scheme for a smart home network. The proposed scheme uses heterogeneous sensor nodes to form a distributed network. In the proposed scheme, the mobile sink and static nodes detect the presence of obstacles through Infra-Red (IR) signal coupling. Also, static sensor nodes transmit data to mobile sinks through ZigBee. The mobile sink moves along the shortest path to collect data from deployed static sensor nodes. The work has clear applications to intelligent pervasive mobile devices including robotic vacuum cleaners and security robots.

The rest of the paper is organized as follows. Section II reviews the related work concerning various obstacle detection strategies in WSNs. Section III presents the system description derived from this research work. Section IV presents the IR based obstacle detection system design. Section V presents the proposed mobile sink based obstacle avoidance scheme for home automation system. Section VI presents the obstacle free path construction scheme. The obstacle avoidance optimal path construction scheme is discussed in Section VII. Experimental results are presented in Section VIII and Section IX concludes this paper.

\section{Related Works}

This section reviews the related literature on static sink based data routing schemes in obstacle resilient WSN.

Stojmenovic and Lin [12] proposed the Greedy Face Greed (GFG) technique for a data routing process with obstacle avoidance. The GFG routing technique reduces the routing path length with the help of nearest neighbor node selection. In the presence of obstacles, GFG forwarding nodes are applied to the right hand neighbor node selection rule for obstacle avoidance path discovery. With the help of the right hand rule, the forwarding node selects suitable neighbor nodes as a next forwarding node in a counter clockwise fashion. 
However, this technique is unable to detect the concave region of an obstacle. Karp and Kung [13] proposed the Greedy Perimeter Stateless Routing (GPSR) technique for obstacle avoidance data transmission. The GPSR technique can avoid static obstacles based on right hand forwarding node selection mechanism. These two techniques can detect the obstacles that are present in the WSN and then successfully transmit sensed data to the BS. However, the GFG and GPSR algorithms cannot discover the energy efficient shortest data routing path due to the lack of global obstacle information.

Zou et al. [14] proposed a distributed algorithm, termed Partial-partition-Avoidance GEographic Routing (PAGER). The proposed technique establishes a shadow region for any concave obstacles. The PAGER technique assigned a cost for each node outside of the shadow region with respect to the distance from node to the sink. The smallest node distance was assigned the smallest cost. Likewise, a higher cost was assigned to the closest sink within a shadow region. Each routing node forwarded received packets to its lowest cost neighbor nodes. In this technique, if any sensor node exhausts its energy within the shadow area, all sensor nodes need to regulate their costs through a routing path update. Therefore, the cost maintaining process of nodes increases control overhead within the network. The PAGER technique detects concave region of the obstacle through the involvement of a large number of nodes. As a consequence energy loss and latency are particularly high in the PAGER algorithm. However, PAGER cannot detect multiple obstacles within the monitoring region.

Chang et al. [15] proposed two route guiding protocols, S-RGP and M-RGP that could detect large size obstacles which are present in the monitoring area. The S-RGP and M-RGP maintain obstacle boundary nodes' information and transmit packets to the shortest path. The S-RGP technique detects obstacle border nodes which are mainly responsible for shortest data routing path construction. The S-RGP technique is used for single obstacle detection and M-RGP is used for multiple obstacle detection. The main drawback of these two techniques is that they require a significant control overhead for detecting a minimum number of boundary nodes. Besides, these two techniques require considerable time to detect shorter boundary path for obstacle avoidance path construction.

Chai et al [8] proposed a sensor network based localization algorithm for an indoor service robot in order to improve localization for the service robot. The authors used a localization algorithm to manage the position data for the fusion sensor agent. Song et al [16] designed and implemented a surveillance robot with hopping capabilities for home security. They used mobile video sensor nodes and ZigBee based wireless communication to design a home control network. Cheng et al [17] proposed a practical path planner scheme for the robotic vacuum cleaner in rectilinear environments. The authors considered two main issues in planning an effective path for a robotic vacuum cleaner, (1) providing a human friendly path and (2) having a low computational load.

The existing static sink based obstacle avoidance techniques can detect obstacles through signal attenuation in the WSN. The energy loss is restrictive which unfortunately degrades network performance. Furthermore, Radio Frequency (RF) signal based obstacle detection schemes cannot detect small size obstacles. Therefore, RF signal based obstacle detection schemes are not ideal for low-power smart home networks.

\section{System Description}

Fig. 1. depicts the proposed automatic smart home monitoring system for this research which consisted of static sensor nodes and a robot-type mobile sink. Static sensor nodes (1..9) are scattered in different locations in the home. These nodes collect data from the monitoring environment and then transmit data to the mobile sink. The mobile sink moves along different paths by avoiding obstacles and collects data from the static sensor nodes to transmit to the BS. Static sensor nodes are coupled to each other through IR based signal transmission for detecting the presence of obstacles between them. Similarly, the mobile sink also transmits an IR signal to the static sensor nodes for detecting the presence of obstacles on its moving path. Each static node detects the emitted IR signal. The BS is used to send the sensed information to another networks. The Zigbee standard is used for communication between the static nodes and the mobile sink. 




Fig. 1. The proposed home monitoring layout.

The main goal of this research was to detect obstacle avoidance by forming an optimal sink movement path under a smart home network. The optimal sink moving scheme can efficiently improve network performance and lifetime. However, another goal is to design a mobile sink based obstacle avoidance routing scheme, which can maximize the reliability of the network.

\section{IR Based Obstacle Avoidance Detection}

In IR based obstacle detection systems, each sensor node couples with its neighbor nodes through IR transmission for the obstacle detection. If any sensor node is unable to detect the IR signal from its neighbor node, it considers that there is the presence of obstacle between them. Once an obstacle has been detected, the node transmits this information to the BS and in this work ZigBee is used. Detailed description of both the static sensor node design and the mobile sink system is described below.

\section{A. Static Sensor Node Design}

Static nodes are designed using a simple 8-bit microcontroller with a ZigBee RF baseband and protocol stack for node-to-node communication. An IR emitter is used for infrared coupling. A local timer is used for generating accurate time delays and additional terminals are provided for triggering or resetting events. A PIN diode and preamplifier are assembled on lead frame. The epoxy package is designed as an IR filter. The demodulated output signal can be directly decoded by the microprocessor.

\section{B. Mobile Sink Design}

Fig. 2. depicts the mobile sink used in this research. The mobile sink contains a robotic based chassis, a motor board, static sensors and an IR based transceiver. The mobile sink movement is controlled by the motor board according to the path information. 




Fig. 2. The proposed mobile sink - a classical vehicular robot design.

\section{Mobile Sink Based data Collection under an Obstacle Resilient Home Network}

This section briefly describes proposed data collection scheme under obstacles resisting home environment. The flow diagram of the proposed scheme is shown in Fig. 3.

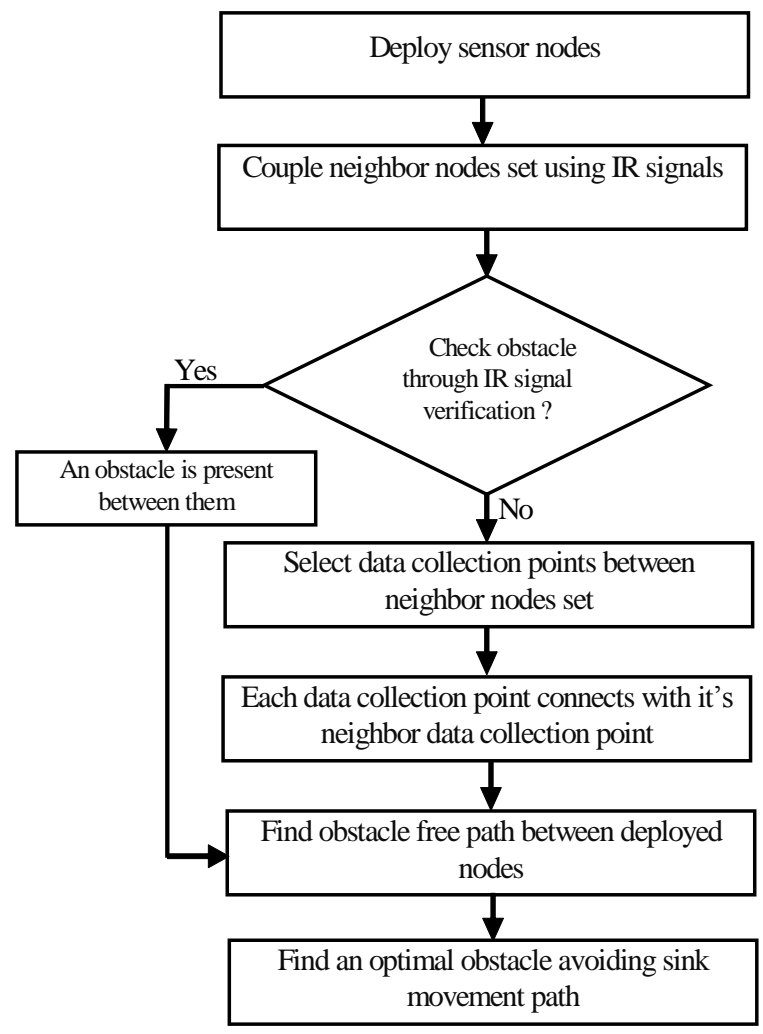

Fig. 3. Flow chart of obstacle free sink movement scheme. 
Each sensor node from deploying node set $S=\left\{s_{1}, s_{2}, \ldots ., s_{n}\right\}$, where $1 \leq n$ is coupled with its single-hop neighbor node set via IR signal transmission. If node $s_{i}$ successfully completes coupling between its single-hop neighbor node set within a specific time period then node $s_{i}$ has not found any obstacles within its neighbors region. Likewise, if node $s_{i}$ is unable to couple with its any neighbor node $s_{j}$ within the allocated time, then it is assumed that node $s_{i}$ has detected an obstacle between $s_{i}$ and $s_{j}$. After the obstacle detection phase, each sensor node finds a common data collection point between its single-hop neighbor nodes set. The mobile sink visits each neighbor node under this data collection point and collects data from each static sensor node. The proposed home network is fully covered by static sensor nodes. Each data collection point is connected to its neighbor data collection point via an obstacle free path.

Fig. 4. shows data collection points between single-hop neighbor nodes set. Similarly, Fig. 5. shows the obstacle free path between the data collection points. In Fig. 5., $\left\{s_{1}, s_{2}, \ldots ., s_{n}\right\}$ sensor nodes find the optimal data collection point $\left\{p_{1}, p_{2}, \ldots . ., p_{n}\right\}$ between them. Each data collection point presents common intersection points of the neighbor node set. During the data collection tour, the mobile sink visits all data collection points to collect data from each node with the objective of optimizing the data collection path length between all data collection points. The optimal data collection path not only increases the network lifetime but also reduces the data transmission delay.

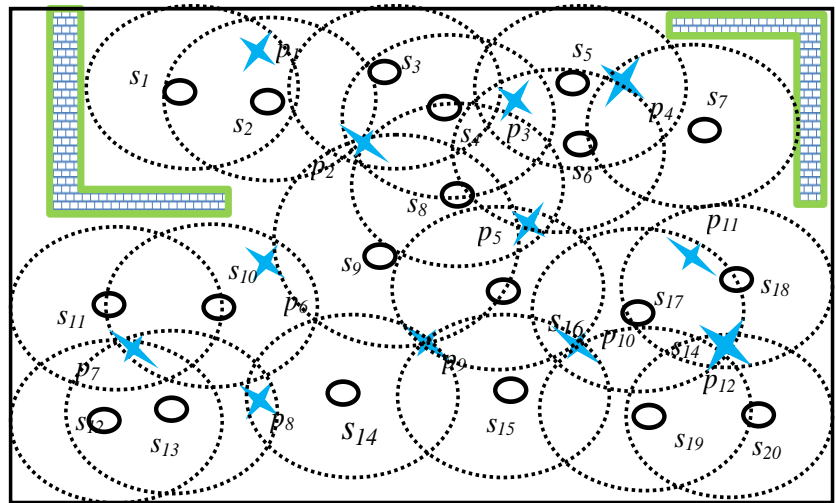

Fig. 4. Data collection points between the neighbor nodes set.



Fig. 5. Existing paths between data collection points.

\section{Obstacle Free Path Discovery Process}

This section discusses the problem of finding the optimal obstacle avoidance sink movement path. The mobile sink moves at a fixed speed along the optimal obstacle avoidance path and collects data from each static sensor node before transmitting data to the BS.

The deployed sensor nodes set, $S$, and its corresponding data collection points $P=\left\{p_{1}, p_{2}, \ldots . ., p_{n}\right\}$, are connected to each other such that any sensor nodes directly communicate to at least one other node. Consider that the 
proposed home network is fully covered by $n$ deployed static sensor nodes. Thus, at least one connection path exists between any $p_{i}$ and $p_{j}$ neighbor data collection points. Similarly, each $p_{i}$ member point covers a subset of $S$ nodes through $k_{i}$ number of coverage demand. Let $F_{i}$ be the fixed cost associated with visiting nodes under any data collection $p_{i}$, and a solution is feasible if each node $p_{i}$ is covered at least $k_{i}$ time by the data collection tour. The primary goal is to minimize the total cost being the sum of the tour length and the fixed cost associated with visiting each node. In the tour, $p_{l}$ denotes starting and ending data collection point of the obstacle avoidance tour. The tour planning process finds a set of data collection points and determents the optimal path to visit them, such that every sensor in $S$ belongs to at least one data collection point within the $P$ set and the total length of the line segments connecting all $P$ members is minimized. In the proposed scheme, WSN defines a complete directed graph $G=(P, A)$ and associates a non-negative cost $c_{i j}$ with each arc, where $c_{i j}$ is equal to the cost of the distance between the candidate data collection points $p_{i}$ and $p_{j}$. The obstacle avoidance minimum data collection tour finding problem can be formulated as follows:

Minimize $\sum_{(i, j) \in A} c_{i j} x_{i j}+\sum_{i \in P} F_{i} m_{i}$

subject to

$$
\begin{array}{lc}
\sum_{j:(i, j)} x_{i j}=\sum_{j:(i, j) \in A} x_{i j}=m_{i}, & \forall i \in P \\
\sum_{j \in P} a_{i j} m_{j} \geq k_{i}, & \forall i \in P \\
\sum_{l \in S} \sum_{k \in P / S} x_{l k}+\sum_{k \in P} \sum_{l \in S} x_{k l} \geq 2\left(m_{i}+m_{j}-1\right) & \\
S \subset P, 2 \leq|S| \leq n-2 & \\
x_{i j} \in\{1,0\} & \forall(i, j) \in A \\
m_{i} \in\{1,0\} & \forall i \in P
\end{array}
$$

where,

$$
\begin{aligned}
& x_{i j}= \begin{cases}1, & \text { if } s_{j} \text { can cover node } s_{i} \text { through obstacle free path } \\
0 & \text { otherwise }\end{cases} \\
& m_{i}= \begin{cases}1, & \text { if node } s_{i} \text { is on the tour } \\
0 & \text { otherwise }\end{cases} \\
& x_{i}=\left\{\begin{array}{cc}
1, & \text { if } a_{i j} \text { is chosen } \\
0 & \text { otherwise }
\end{array}\right.
\end{aligned}
$$

Objective function (1) minimizes the total cost of the obstacle free data collection tour and sensor node visiting cost. Constraint (2) ensures the fact that every no-tour data collection point has one incoming and outgoing arc. Constant (3) specifies that each sensor node must be visited by the mobile sink in the tour. Constant (4) is a connectivity constraint that ensures that there are no sub-tours. Constraint (5) and constraint (6) define the variables as binary. 


\section{Obstacle Avoidance Optimal Path Construction Scheme}

The obstacle avoidance sink movement problem is an NP-hard problem. This work develops a heuristic algorithm to solve the above obstacle avoidance sink movement problem approximately. This research has related the obstacle free sink movement problem with a similar Covering Salesman Problem (CSP). Current and Schilling [18] proposed the CSP for tour planning between a sub set of all cities and they also prove that CSP is an NP-hard problem. In the home environment with obstacles, the mobile sink visits each data collection point that covers a subset of deployed nodes and collects data from the sensor node. Therefore, the optimal path construction problem in a home environment with obstacles is an NP-hard problem. Current and Schilling [18] also suggested a heuristic solution for CSP. In this heuristic solution, CSP defines two sub problems. The first solution finds the minimum number of vertex covers by salesman while the second solution determines the shortest tour between them.

The main idea of this proposed greedy algorithm is to choose a set of data collection points with respect to the deployed node set. Each data collection point corresponds to a neighbor set of sensor nodes. In each step, a neighbor set of sensor nodes can be covered when its corresponding data collection point is chosen in the data collection tour. If all sensor nodes are covered by the data collection point set, the algorithm is terminated. This algorithm finds a path to cover each uncovered neighbor set of sensors with the minimum average cost. In the proposed greedy algorithm, $P_{\text {current }}$ contains all data collection points, $S$ contains all deployed sensor nodes that transmit data to mobile sink, and $U_{\text {current }}$ contains the set of all remaining uncovered sensor nodes at each step. In every tour, the mobile sink visits each data collection point at least once to collect data from a subset of the static nodes. Initially, $P_{\text {current }}$ is empty i.e. no data collection point has been selected. Each $s_{i}$ node finds a data collection point $p_{i}$ between its single-hop neighbor node set $n_{l}\left(s_{i}\right)$ through average cost calculation. Single-hop neighbor nodes under $p_{i}$ data collection points are visited by the mobile sink. After data has been collected from the neighbor node set then the mobile sink moves to the next data collection point $p_{j}$ which is selected from $U_{\text {current }}$. Let $\theta=\operatorname{cost}\left\{n_{1}\left(s_{i}\right) /\left(n_{1}\left(s_{i}\right) \cap U_{\text {current }}\right)\right\}$, which denotes the average cost to cover all uncover sensor in $n_{l}\left(s_{i}\right)$. While there are remaining uncovered elements in $U_{\text {current }}$, the uncovered nearest second-hop neighbor set $n_{2}\left(s_{i}\right)$ is chosen with the minimum $\theta$ value, add the corresponding data collection point $p_{i}$ of $n_{l}\left(s_{i}\right)$ into $P_{\text {current }}$, and remove the corresponding covered sensor from $U_{\text {current }}$. The algorithm terminates when all nodes are covered and formally described in Algorithm I. Finally, $P_{\text {current }}$ contains all data collection points in the datagathering tour. After covering all the deployed sensor nodes, the data collection tour can be easily conducted between the $P_{\text {current }}$ set member points. The optimal tour between all $P_{\text {current }}$ member points is easily obtained by running any approximate algorithm for the TSP.






\section{Experimental Results}

Fig. 6(a). depicts the experimental setup for this research. Nine static sensors are deployed into three rooms for the home environment monitoring. One robotic based mobile sink is used for data collection.

\section{A. Environmental Setup}

The experiment tests the feasibility of the proposed scheme on real life implementation using a mobile robotic vehicle (Fig. 2.) emulating such devices as security robots or robotic vacuum cleaners. In the experiments, all static sensor nodes are initially the same and each sensor node communicates to its neighbor nodes through single-hop communication. Each node verifies the presence of an obstacle through IR signal coupling. Each static sensor periodically transmits an IR signal to it's neighbor nodes for obstacle verification. If any obstacle presents between the nodes, IR signals are unable to reach neighboring nodes. In that condition, the node detects an obstacle between them.

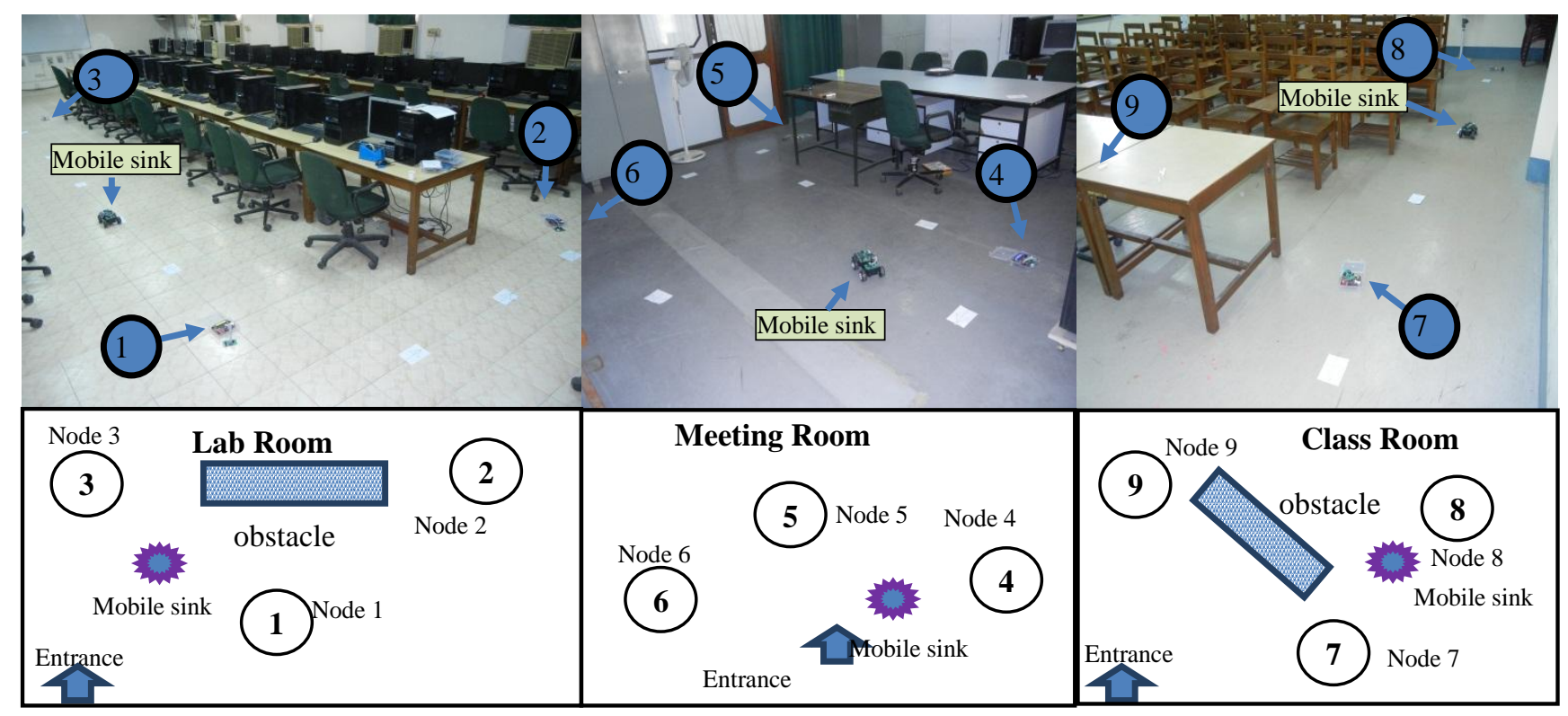

(a): Mobile sink based data collection experiment, static sensor nodes are deployed in three rooms (Lab room, Meeting room, and Class room), and these three rooms are connected through a common corridor.



(b): Data collection point set and all possible paths between them (through simulation). 


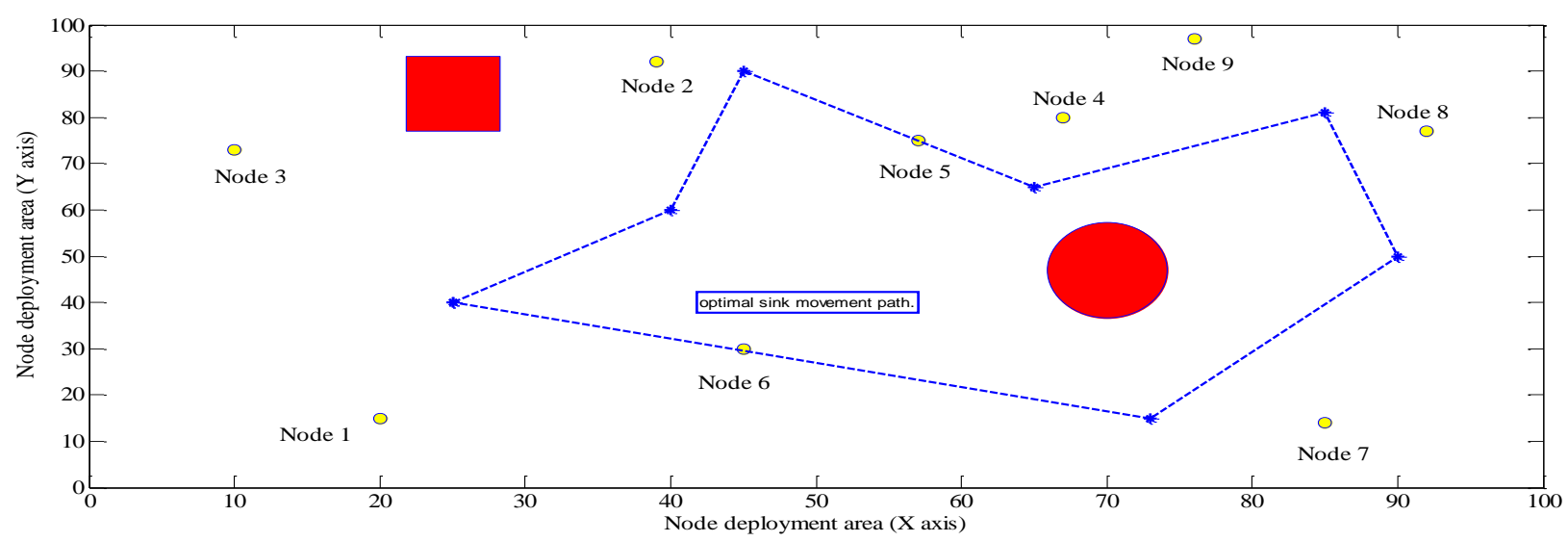

(c): Optimal sink mobility path (after optimization).

Fig. 6. Implementation and simulation of the proposed smart home monitoring network.

\section{B. Experimental Results}

Experiments were designed and carried out, one computer based office, one meeting room and one classroom. Static sensor nodes (S1, S2, and S3) are deployed in the room (Fig. 6(a).). Within the room, the mobile sink directly collected the data from $\mathrm{S} 1, \mathrm{~S} 2$, S3. In this room only two data collection points have been detected by $\mathrm{S} 1, \mathrm{~S} 2$, and S3 sensor nodes due to the presence of obstacles, S4, S5, and S6 nodes are deployed within the meeting room. In this room, S4, S5 and S6 nodes detect a single data collection points between them and transmit data to mobile sink. Similarly, S7, S8, and S9 sensor nodes are deployed in the classroom. In this room, two data collection points are detected between S7, S8, and S9. Fig. 6(b) shows the data collection point set and paths between them. The optimal path for mobile sink movement is shown in Fig. 6(c). Fig. 7. shows the path cost found between the data collection points.

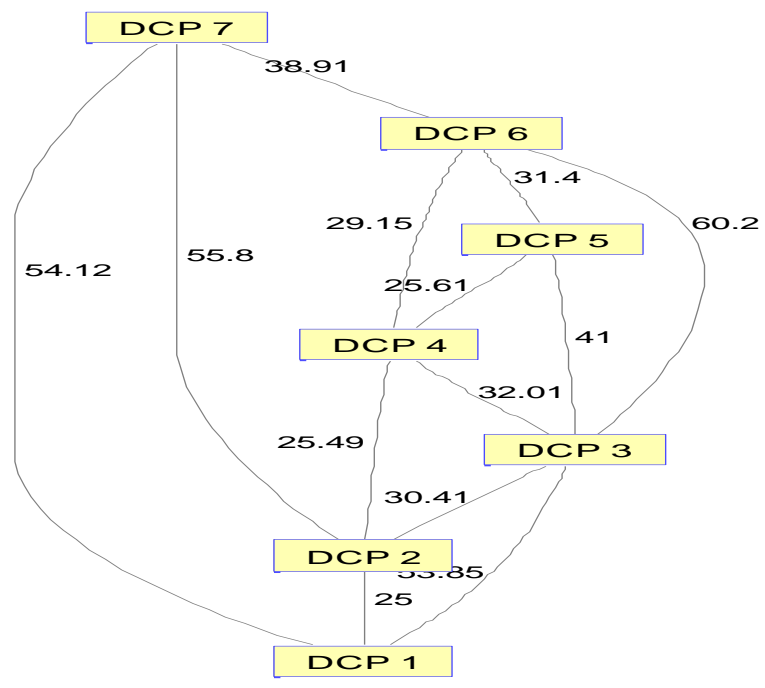

Fig. 7. All paths between the Data Collection Point (DCP) and cost. 
Fig. 8. and Fig. 9. depict the measured temperature and light intensity results by the sensor nodes. The lab and classroom temperature are regulated by an air-conditioner and verified with actual sensed information. S4, S5, and S6 nodes sense the light condition of the meeting room.

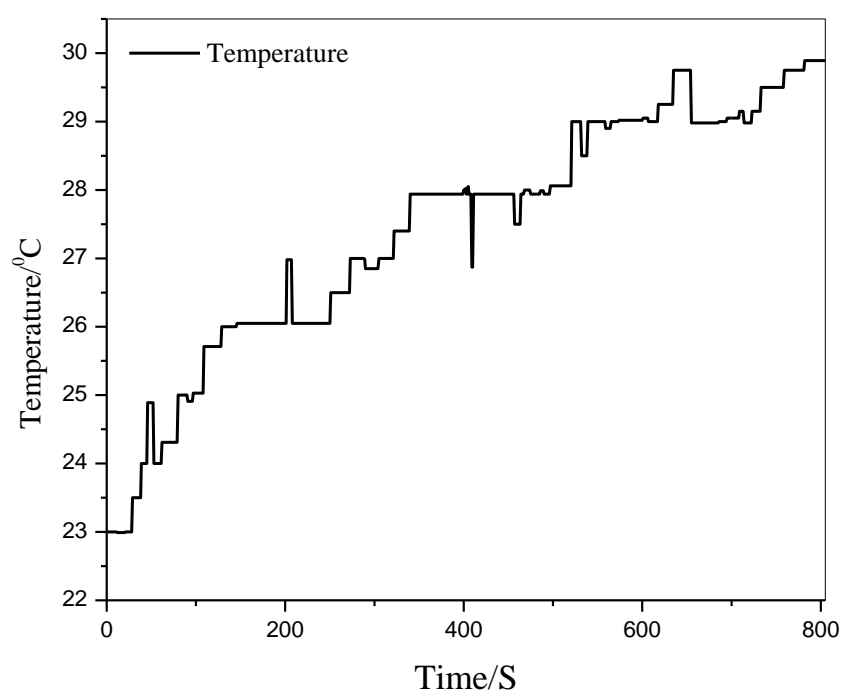

Fig. 8. Indoor environment monitoring excremental result (Temperature).



Fig. 9. Indoor environment monitoring excremental result (Light intensity).

Fig. 10. shows the total tour length travelled by the mobile sink as a function of the number of obstacles. As the number of obstacles increases then as expected the tour length required also increases because mobile sink needs to find an alternate path for data collection.

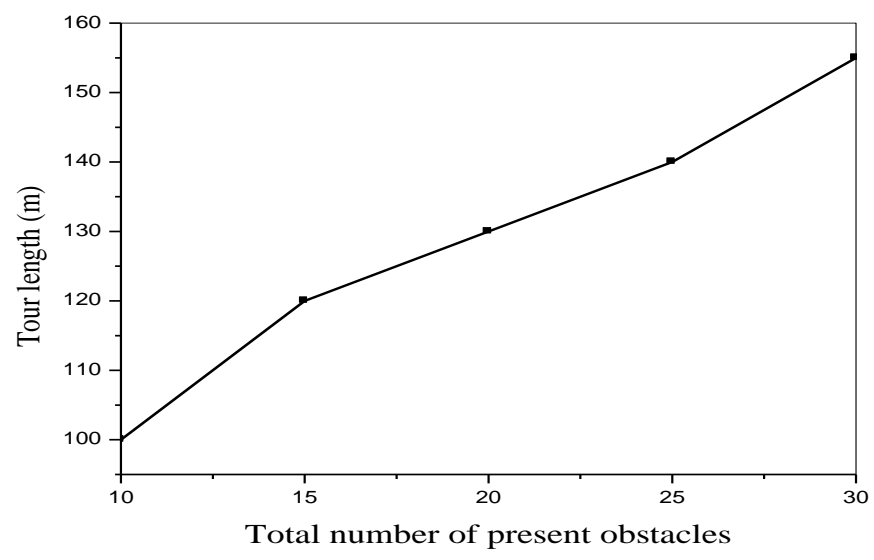

Fig. 10. Total tour length versus total number of present obstacles. 
The total energy consumption of the proposed scheme and the M-RGP scheme [15] (in units of Joule) is depicted in Fig. 11. As can be seen, the proposed scheme derived from this research performs significantly better compared to the M-RGP scheme primarily due to the optimal sink mobility that reduces the energy loss of the static sensor nodes.



Fig. 11. Total energy loss comparison.

Fig. 12. shows the network lifetime comparison results between the static sink based data collection scheme and the mobile sink based data collection scheme. The proposed sink mobility based data collection scheme has resulted in an increased network lifetime of $30 \%$ compared to the static sink based data collection scheme. Therefore, the network lifetime is increased as a result of using the mobile sink based data collection scheme.

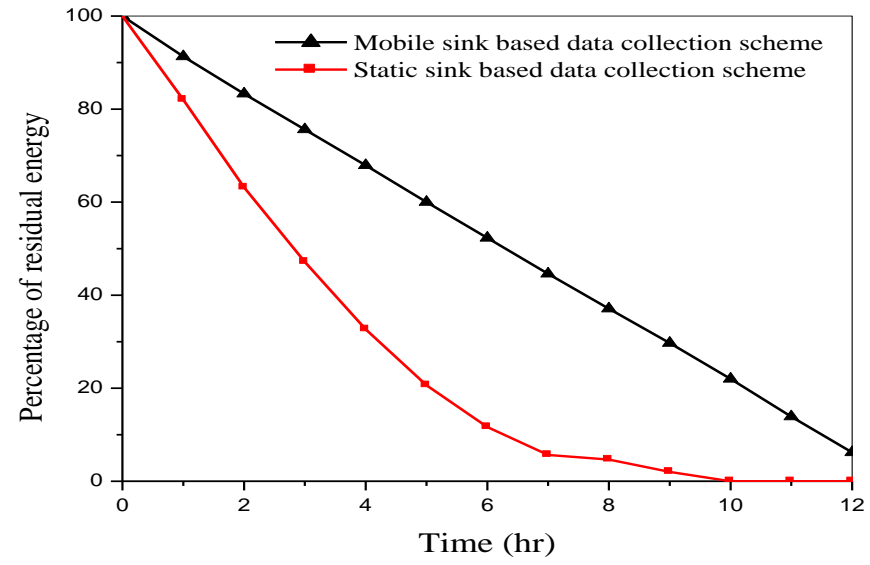

Fig. 12. Network lifetime comparison. 


\section{Conclusion}

Recently sink mobility has been playing an increasingly important role in the environmental design of modern smart homes. This paper proposes a mobile sink based obstacle avoidance routing scheme for smart home monitoring systems. In the research, the deployed sensor nodes can detect the presence of obstacles between them through IR communication and improve the transmission of data to the mobile sink. Furthermore, the mobile sink implements obstacle avoidance to find the shortest path to collect data from static deployed sensor nodes.

The proposed scheme is implemented through ZigBee enabled hardware and prototyped in realistic environments in order to validate the performance. By using the results from this research, the network lifetime can be 30\% longer than using previous static sink based data collection schemes. The application of this work is aimed at intelligent pervasive consumer products and services including robotic vacuum cleaners and personal security robots.

\section{References}

[1] H. Oh, H. Bahn, and K.-J. Chae, "An energy-efficient sensor routing scheme for home automation networks," IEEE Trans. Consum. Electron., vol. 51, no. 3, pp. 836-839, Aug. 2005.

[2] W.-K. Park, I. Han, and K.-R. Park, "ZigBee based dynamic control scheme for multiple legacy IR controllable digital consumer devices,” IEEE Trans. Consum. Electron., vol. 53, no. 1, pp. 172-177, Feb. 2007.

[3] L. Cheng, C.-D. Wu, and Y.-Z. Zhang, "Indoor robot localization based on wireless sensor networks," IEEE Trans. Consum. Electron, vol. 57, no. 3, pp. 1099-1104, Aug. 2011.

[4] I. A. Zualkernam, A. R. Al-Ali, M. A. Jabbar, I. Zabalawi, and A. Wasfy, "InfoPods: zigbee-based remote information monitoring devices for smart-homes, " IEEE Trans. Consum. Electron., vol. 55, no. 3, pp. 1221-1226, Aug. 2009.

[5] I. Banerjee, P. Chanak, H. Rahaman, and T. Samanta, "Effective fault detection and routing scheme for wireless sensor networks, " Computers \& Electrical Engineering, Elsevier, vol. 40, no. 2, pp. 291-306, Feb. 2014.

[6] J. Wang, Y. Yin, J. Zhang, S. Lee, and R. S. Sherratt, "Mobility based energy efficient and multi-sink algorithms for consumer home networks," IEEE Trans. Consum. Electron., vol. 59, no. 1, pp. 78-84, Feb. 2009.

[7] S. Kim, J. Y. Sim, and S. Yang, "Vision-based cleaning area control for cleaning robots," IEEE Trans. on Consum. Electron., vol. 58, no. 2, May 2012.

[8] B. S. Choi and J.-J. Lee, "Sensor network based localization algorithm using fusion sensor-agent for indoor service robot," IEEE Trans. on Consum. Electron., vol. 56, no. 3, pp. 1457-1465, Aug. 2010.

[9] K. Gill, S. H. Yang, F. Yao, and X. Lu, "A zigbee based home automation system," IEEE Trans. Consum. Electron., vol. 55, no. 2, pp. 422-430, May. 2009.

[10] S. Kim, J. S. Kang, H. S. Park, Y.-J. Kim, and D. Kim, "UPnP-zigbee internetworking architecture mirroring a multihop zigbee network topology,” IEEE Trans. Consum. Electron., vol. 55, no. 3, pp. 1286-1294, Aug. 2009.

[11] H. S. Kim, J.-H. Song, and S. Lee, "Energy-efficient traffic scheduling in IEEE 802.15.4 for home automation networks," IEEE Trans. Consum. Electron., vol. 53, no. 2, pp. 369-374, May. 2007.

[12] I. Stojmenovic and X. Lin, "GEDIR: loop-free location-based routing in wireless networks, " in Proc. 11th IASTED Int. Conf. on Parallel and Distributed Computing and Systems, Boston, MA., pp. 1025-1028, Nov. 1999.

[13] B. Karp and H. T. Kung, "GPSR: Greedy perimeter stateless routing for wireless networks," in Proc. 6th ACM Annu. Int. Conf. Mobile Comput. Boston, MA., pp. 243-254, Aug. 2000.

[14] L. Zou, M. Lu, and Z. Xiong, "A distributed algorithm for the dead-end problem of location-based routing in sensor networks," IEEE Trans. Veh. Technol., vol. 54, no. 4, pp. 1509-1522, July 2005.

[15] C.-Y. Chang, C.-T. Chang, Y.-C. Chen, and S.-C. Lee, "Active route-guiding protocols for resisting obstacles in wireless sensor networks," IEEE Trans. Veh. Technol., vol. 59, no. 9, pp. 4425-4442, Nov. 2010.

[16] G. Song, K. Yin, Y. Zhou, and X. Cheng, "A surveillance robot with hopping capabilities for home security," IEEE Trans. Consum. Electron, vol. 55, no. 4, pp. 2034-2039, Nov. 2009.

[17] L. Cheng, C.-D. Wu, and Y.-Z. Zhang, "Indoor robot localization based on wireless sensor networks," IEEE Trans. Consum. Electron, vol. 57, no. 3, pp. 1099-1104, Aug. 2011.

[18] J. R. Current and D. A. Schilling, "The covering salesman problem,” Transp. Sci., vol. 23, no. 3, pp. 208-2013, Aug. 1989. 


\section{Biographies}



Prasenjit Chanak (S'12) received the B.Tech degree in information Technology for the Institute of Engineering and Technology (IET), Jaunpur, India followed by the M.Tech degree in information technology from Bengal Engineering and Science University (BESU), Shibpur, West Bengal, India and is currently working toward the Ph.D. degree in Information Technology at Indian Institute of Engineering Science and Technology (IIEST), Shibpur, West Bengal, India.

He is the recipient of a senior research fellowship (SRF), CSIR, Govt. of India, His research interests are in the areas of wireless ad hoc and sensor networks and wireless and mobile systems. He is a member of the IEEE Sensor Council.

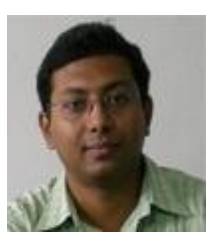

Indrajit Banerjee (M'11) received the Bachelor degree in Mechanical Engineering from Institute of Engineers, India, the Masters in Information Technology from Bengal Engineering and Science University, and the PhD. in Information Technology from Indian Institute of Engineering Science and Technology (IIEST), Shibpur, India.

$\mathrm{He}$ is currently an assistant professor in the information technology department at the Indian Institute of Engineering Science and Technology (IIEST), Shibpur, India. His current pervasive computing.

interests are cellular automata, wireless ad hoc and sensor network, embedded systems and

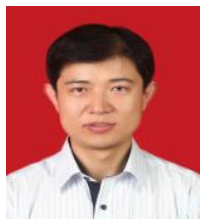

Jin Wang (M'11) received the B.S. and M.S. degree from Nanjing University of Posts and Telecomm., China in 2002 and 2005, respectively. He received Ph.D. degree from Kyung Hee University Korea in 2010. Now, he is a professor in the Computer and Software Institute, Nanjing University of Information Science and Technology.

He has published more than 160 journal and conference papers. His research interests mainly include routing protocol and algorithm design, performance evaluation and optimization for wireless ad hoc and sensor networks.

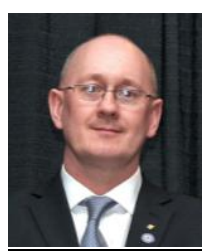

R. Simon Sherratt (M'97-SM'02-F'12) received the B.Eng. degree in Electronic Systems and Control Engineering from Sheffield City Polytechnic, UK in 1992, M.Sc. in Data Telecommunications in 1994 and Ph.D. in video signal processing in 1996 from the University of Salford, UK.

In 1996, he has appointed as a Lecturer in Electronic Engineering at the University of Reading where he is now a Professor of Consumer Electronics and Head of Wireless and Computing research. His research topic is signal processing in consumer electronic devices.

Eur Ing Professor Sherratt was an IEEE Consumer Electronics Society Vice President (08-09) and a serving AdCom member (03-08, 10-15). He received the IEEE Chester Sall Memorial Award in 2006 and is now the Editor-in-Chief of the IEEE Transactions on Consumer Electronics. 